\title{
Efectos del desempleo en las relaciones de pareja, en el ambiente familiar y en la salud mental
}

Andrea Parejo Ortega, Rocío Sánchez, Andrea Vázquez Gil e Irene Valero Pizarro. Universidad de Granada

Recepción: 12 de mayo de 2015 | Revisión: 17 de junio de 2015 | Aceptación/Publicación: 17 de julio de 2015

Citar: Parejo, A., Sanchez, R., Vazquez, A. y Valero, I. (2015). Efectos del desempleo en las relaciones de pareja, en el ambiente familiar. ReiDoCrea, 4, 106-111.

Resumen: El objetivo del presente estudio es analizar los efectos del desempleo en las relaciones de pareja, en el ambiente familiar y en la salud mental. Para ello se han tenido en cuenta variables como la edad, el sexo, el número de hijos y el tiempo de relación. Para llevar a cabo la presente investigación se ha llevado a cabo con una muestra de 70 personas (35 parejas heterosexuales). Este grupo de sujetos se reparte en cuatro condiciones: ambos empleados, mujer desempleada, hombre desempleado y ambos desempleados. Perteneciendo a cada grupo 12 parejas, 8 parejas, 6 parejas y 9 parejas, respectivamente. Las conclusiones de este estudio indican que la situación laboral no influye ni en las relaciones de pareja ni en el ambiente familiar. Sin embargo, se encuentran efectos significativos en la salud mental.

Palabras clave: Desempleo, Salud mental, Familia, Recesión económica

Unemployment's effects in relationships, in the family atmosphere and in the mental health

Abstract: The object of the present study is to analyze the unemployment's effects in the relationships, in the family atmosphere and in the mental health. For this goal we have considered the next variables: age, gender, number of children and relationship time. We have done the investigation with a sample of 72 people (36 heterosexual couples). This sample is distributed in four conditions: Both unemployed, woman unemployed, man unemployed and both unemployed. Each group had 12 couples, 8 couples, 6 couples and 9 couples, respectively. The findings of this study indicate that the employment situation does not affect the relationships or the family atmosphere. However, there are significant effects on mental health.

Key words: Unemployment, Mental health, Family, Economic recession.

\section{Introducción}

El desempleo aparece como psicológicamente destructivo, incluso en aquellos casos en que están garantizados los ingresos económicos (Buendía, 1990). Esto se debe en buena parte a que representa la ruptura de un sistema de relaciones (compañeros de trabajo, gente que trabaje en el lugar...), y como consecuencia de todo ello la situación de desempleo llega a perturbar al individuo provocando en él sentimientos de inferioridad ante la pérdida de autoestima y la minoración del status social. (Buendía, 1990). Además la incertidumbre acerca de la situación laboral futura actúa como un factor estresante que conduce a cambios psicológicos, que también van a impactar negativamente en la salud mental (Urbanos-Garrido y González, 2013).

Las personas desempleadas presentan un riesgo entre dos y siete veces mayor de padecer depresión que las personas que están empleadas. Asimismo, el desempleo aumenta de manera significativa los trastornos de ansiedad, los somatomorfos, y el abuso de alcohol (Gill, Roca, y García-Campayo, 2014).

El desempleo tiene más efectos negativos en la salud mental en el hombre que en la mujer, debido a que las responsabilidades de principal proveedor de la familia se suelen atribuir al hombre (Artazcoz, Benach, Borrell, y Cortés, 2004). La estabilidad del hogar siempre ha girado en torno a la ocupación del varón, y por tanto, cuando es el 
hombre el que pierde el trabajo, la propia relación tiene muchas más dificultades de mantenerse como estaba. El hombre que tiene que llevar la casa siente malestar, ya que su desempleo le coloca en una dependencia salarial de su mujer, lo que resulta duro para su autoestima. Sin embargo, cuando el paro afecta a la mujer, se reestablece una normalidad que estaba latente y entra en un cierto sentido de lo razonable (Briales, 2013).Los hombres que tienen una relación se preocupan más por cumplir su papel masculino frente a la sociedad. De esta manera, los hombres desempleados que lleven menos de 3 años en la relación de pareja experimentarán más malestar que aquellos que llevan más años (García y Nader, 2009).

El desempleo no va a afectar únicamente a la persona desempleada, sino también a los miembros de su familia, de manera que la percepción de problemas económicos por parte de los niños afecta directamente a la presencia de problemas de ansiedad y de estado de ánimo en los niños (Guillamón, 2003). Igualmente, el despido laboral podría estar implicado en un aumento de las discusiones maritales y un decremento en el nivel de apoyo, implicación y afecto que los progenitores dispensan al niño (Guillamón, 2003).Aparte de esto, la disminución de ingresos y otros factores producen cargas extras de trabajo doméstico y de cuidados que van a repercutir negativamente en las relaciones domésticas (Briales, 2013). En los últimos años se ha podido ver un cambio en la mujer del modelo caregiver(cuidadora) al dual breadwinner/caregiver (sustentadora/cuidadora); siguen siendo ellas las que afrontan los cuidados y esfuerzos familiares. Se les exige más y se les entrega menos (De Lucas y De la Cueva, 2009).

Por otro lado, en el caso de las parejas de doble empleo, estas pueden experimentar un conflicto entre el ámbito familiar y laboral o bien una facilitación (Aguirre y Martínez, 2006).Estudios previos han vinculado la acumulación de estresores con dificultades en el desempeño exitoso del rol parental, generando consecuencias negativas para el desarrollo de los menores y un mal ambiente familiar. Estos estresores son principalmente la familia (malas relaciones maritales, un hogar inestable, algunas características de los hijos y tensiones constantes) y el trabajo (número de horas dedicadas o la falta de flexibilidad para conciliar la vida familiar). Ser madre o padre de un mayor número de hijos en la familia tiende a aumentar la sensación de estrés parental por sí mismo y es uno de los estresores más significativos (Pérez, Lorence y Menéndez, 2010).

A continuación se van a presentar nuestras hipótesis principales:

- El desempleo causa una peor relación con la pareja en familias en las que ambos están desempleados, en comparación con otras parejas en las que ambos o alguno de los miembros trabaje.

- El desempleo causa efectos negativos en la salud mental, en mayor medida en aquellas parejas en las que ambos miembros están desempleados, en comparación con otras parejas en las que ambos o alguno de los miembros trabaje.

A lo largo de la investigación, han ido surgiendo otras hipótesis que presentamos a continuación:

- Las parejas que lleven más de tres años de relación sufrirán en menor medida los efectos negativos del desempleo en la relación de pareja.

- Las relaciones familiares se verán afectadas negativamente por el desempleo.

- En caso de desempleo a mayor número de hijos la salud mental de sus progenitores se verá empeorada. 


\section{Método y materiales}

Para la realización de este estudio hemos cogido una muestra de 72 personas agrupadas en 36 parejas heterosexuales. Cada pareja pertenecía a un grupo determinado en función de su situación laboral, formándose así cuatro condiciones experimentales. La condición 1 formada por 12 parejas, correspondiente al grupo en el cual ambos miembros de cada pareja estaban empleados. En la condición 2, constituida por 8 parejas, la mujer estaba desempleada. La condición 3, compuesta por 6 parejas, el hombre estaba desempleado. Por último, en la condición 4, compuesta por 9 parejas en la que ambos miembros de la pareja estaban desempleados.

A los participantes se les entregó un cuadernillo compuesto por un consentimiento informado y una serie de cuestionarios a rellenar; Cuestionario de Salud de Goldberg, el cual mide síntomas somáticos, ansiedad insomnio, disfunción social y depresión; Modelo Triangular de Amor de Sternberg, el cual mide intimidad, pasión y compromiso; y Cuestionario de Satisfacción Familiar. Debían rellanarlos independientemente de su pareja, facilitando así, la mayor libertad y sinceridad a la hora de contestar las preguntas.

A cada implicado se le pidió que rellenara una serie de datos demográficos: edad, sexo, tiempo de relación, número de hijos y situación laboral.

\section{Resultados}



En este primer análisis de nuestro estudio se observa, de manera individual, puntuaciones significativas con respecto a la correlación entre situación laboral de los participantes y su salud mental. Sin embargo, tras el análisis de la relación existente entre la situación laboral (los dos trabajando, mujer en desempleo, hombre en desempleo y ambos desempleados) con respecto a la relación que la pareja mantenía no se observan datos significativos que indiquen un relación directa entre la situación laboral y la relación de pareja.

Gráfica 1. Medias de la situación laboral en relación con la salud mental

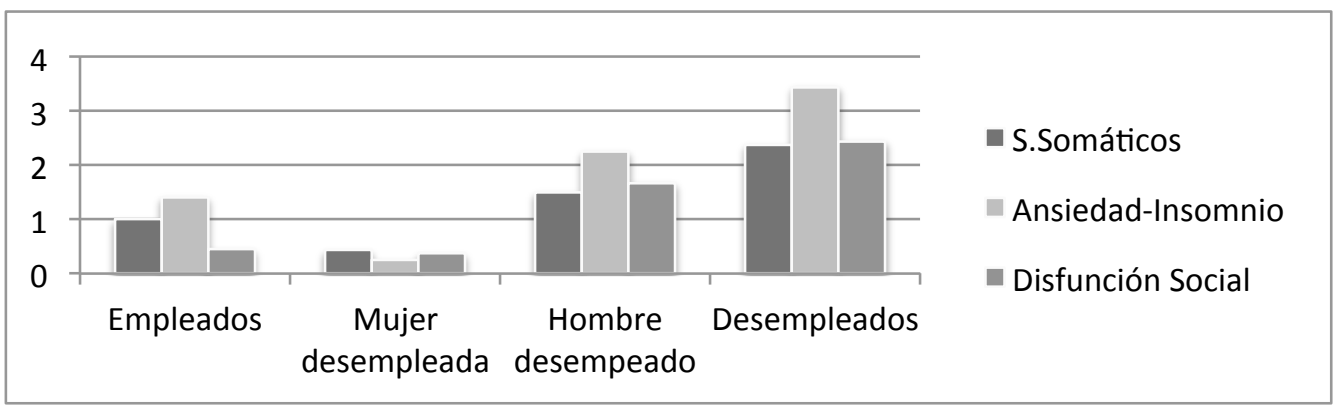


Las puntuaciones medias globales de las cuatro condiciones, en las cuales se distribuían los participantes voluntarios, indicarían una notable diferencia con respecto a nuestras variables a evaluar: síntomas somáticos, ansiedad-insomnio y disfunción social. La condición "desempleados" sería la que peor resultados globales obtiene, con respecto a las otras condiciones, en todas las variables a evaluar. Sin embargo, como datos relevantes en esta gráfica podemos observar: como la condición "mujer desempleada" es la que muestra puntuaciones globales más bajas con respecto a las variables estudiadas con respecto al resto de las condiciones y como, la condición "hombre desempleado" sería después de "desempleados" la condición que peores medias globales obtiene en todas las variables (síntomas somáticos, ansiedadinsomnio y disfunción social). A su vez, la variable ansiedad-insomnio es la que peor media global tiene en las condiciones: empleados, hombre desempleado y desempleados.

\begin{tabular}{|c|c|c|c|c|}
\hline \multicolumn{5}{|l|}{ Anou multifactorial relacionanda situaci } \\
\hline VI & $V D$ & $F$ & $g l$ & $p$ \\
\hline \multirow{3}{*}{ Situación laboral } & Intimidad & 0,403 & 3 & 0,751 \\
\hline & Pasión & 1,378 & 3 & 0,258 \\
\hline & Compromiso & 1,136 & 3 & 0,314 \\
\hline \multirow{3}{*}{ Grupo tiempo $^{a}$} & Intimidad & 1,018 & 1 & 0,317 \\
\hline & Pasión & 3,573 & 1 & 0,063 \\
\hline & Compromiso & 0,297 & 1 & 0,588 \\
\hline \multirow{3}{*}{ Situación laboral *Grupo tiempo } & Intimidad & 0,704 & 1 & 0,405 \\
\hline & Pasión & 1,877 & 1 & 0,175 \\
\hline & Compromiso & 1,603 & 1 & 0,210 \\
\hline
\end{tabular}

A partir de los datos obtenidos en el anova multifactorial se observa, tras la correlación entre las variables: situación laboral con el tiempo en la relación y el efecto en la relación de pareja, que no hay datos significativos que muestren una relación significativa entre éstas. Es decir, no existiría una relación directa entre el tiempo que lleva la pareja y el efecto de la relación de pareja. A su vez, tampoco hay evidencia de que exista una relación directa entre situación laboral con el tiempo en la relación y el efecto en la relación de pareja.

\begin{tabular}{|c|c|c|c|c|}
\hline \multicolumn{5}{|c|}{$\begin{array}{c}\text { Tabla } 3 \\
\text { Anova multifactorial relacionando la situación laboral con el número de hijos y el efecto en la salud mental }\end{array}$} \\
\hline VI & VD & $F$ & $g l$ & $p$ \\
\hline \multirow{5}{*}{ Situación laboral } & Somatización & 4,781 & 3 & 0,005 \\
\hline & Ansiedad-insomnio & 5,709 & 3 & 0,002 \\
\hline & Disfunción social & 4,473 & 3 & 0,007 \\
\hline & Depresión & 0,620 & 3 & 0,605 \\
\hline & Somatización & 0,175 & 1 & 0,677 \\
\hline \multirow{4}{*}{ Grupo tiempo a } & Ansiedad-insomnio & 0,175 & 1 & 0,677 \\
\hline & Disfunción social & 1,6121 & 1 & 0,209 \\
\hline & Depresión & 1,141 & 1 & 0,290 \\
\hline & Somatización & 1,141 & 1 & 0,290 \\
\hline \multirow{3}{*}{ Situación laboral * Grupo tiempo } & Ansiedad-insomnio & 1,092 & 1 & 0,359 \\
\hline & Disfunción social & 0,603 & 1 & 0,615 \\
\hline & Depresión & 1,323 & 1 & 0,275 \\
\hline
\end{tabular}

Las correlaciones realizadas muestran, lo anteriormente visto en las tablas anteriores, que existe una relación directamente proporcional con respecto a la condición laboral y las variables somatización y ansiedad-insomnio. Sin embargo, los datos obtenidos en este anova multifactorial señalan a que no existe relación alguna entre el número de hijos con el resto de las variables (somatización, ansiedad-insomnio, disfunción social 
y depresión) debido a que los resultados obtenidos en estas correlaciones no son significativos.

\section{Discusión}

Para realizar los análisis estadísticos se utilizó el programa de SPSS versión 18.0. Los análisis realizados se centraron en un cálculo de anovas multifactoriales entre las variables objeto de estudio: la situación laboral con la salud mental y las relaciones de pareja, la situación laboral con el tiempo en la relación y el efecto en la relación de pareja, la situación laboral con el número de hijos y el efecto en la salud mental y post hoc.

En vista a los resultados se puede concluir, tal y como exponían Urbanos-Garrido y González (2013), que el desempleo afecta significativamente en una peor salud mental. Esta se ve reflejada en los trastornos somáticos, en la ansiedad-insomnio y en la disfunción social, afectando estas en primer lugar a las parejas en las que ambos miembros se encuentran en desempleo, seguidos del hombre desempleado, confirmándose nuestra segunda hipótesis principal. Sin embargo, se desmiente nuestra primera hipótesis principal, es decir, a la vista de la tabla 1, observamos que el desempleo no causa una peor relación de pareja (la diferencia entre grupos de intimidad, pasión y compromiso no es significativa). Estos resultados no se ven afectados en la interacción con el tiempo de relación de la pareja (tabla 2). Por lo tanto, también se desmiente la hipótesis secundaria de que las parejas que lleven más de tres años de relación sufrirán en menor medida los efectos negativos del desempleo en la relación de pareja, contrariamente a lo expuesto en el artículo de García y Nader (2009) .Respecto al clima familiar, la situación laboral no influye en ésta como indican los resultados de la tabla 1. Consecuentemente, rechazamos la hipótesis secundaria de que las relaciones familiares se verán afectadas negativamente por el desempleo, de forma incongruente a los datos recogidos en la tesis de Guillamón (2003). Por último, en referencia al número de hijos, en la tabla 3 se observa que no hay un efecto significativo entre la situación laboral y la salud mental, por lo que rechazamos nuestra última hipótesis secundaria, que afirmaba que en el caso de desempleo, a mayor número de hijos, la salud mental de sus progenitores se verá empeorada, lo cual es incompatible con los resultados obtenidos en el artículo de Pérez, Lorence y Menéndez (2010).

Curiosamente, podemos observar que la salud mental es peor en las parejas en las que ambos trabajan, que en las parejas en las que la mujer se encuentra en situación de desempleo, trabajando el hombre. Atribuimos estos resultados al rol que siempre se le ha asignado al hombre como proveedor principal de la familia y a la mujer como ama de casa, apoyando la teoría expuesta en el artículo de Briales (2013), que sostenía, que a pesar de que el hombre se ocupa cada vez más de las labores domésticas y de crianza, todavía no se concibe la posibilidad de un padre no proveedor, de manera que cuando el hombre pierde el empleo, la situación se vuelve insostenible, mientras que cuando se trata de la mujer, se vuelve a una "normalidad latente".

Finalmente, tenemos que decir que como limitaciones de este estudio, del cuestionario de satisfacción familiar empleado no se conoce su fiabilidad y validez, puesto que no ha sido sometido al proceso requerido para su comprobación y que la muestra de parejas es relativamente pequeña, por lo que para futuras investigaciones sería conveniente usar un test validado y una muestra más amplia con el fin de corroborar 0 rechazar algunos de los datos obtenidos en este estudio. 


\section{Referencias}

Aguirre Zubiaurre, Z., Martínez Díaz, M.P. (2006). Influencia de la situación laboral en el ajuste familia-trabajo. Fundación Mapfre Medicina, 17(1),

Artazcoz, L., Benach, J., Borrell, C., y Cortés, I. (2004). Unemployment and Mental Health: Understanding the Interactions Among Gender, Family Roles, and Social Class.American Journal of Public Health, 94 (1), 82-88.

Briales, A. (2013). Paro,crisis de los cuidados y relaciones de género: conflictos y ambivalencias. En M. A. García. GT 12 Sociología del género. Madrid: Universidad Complutense de Madrid.

Buendía, J. (1990). Psicopatología del desempleo. Anales de psicología, 6 (1), 21-36.

De Lucas, F., y De La Cueva, M. (2009). Crisis anunciada y sujeción de la mujer en el régimen familiarista. (Tesis doctoral). Universidad Complutense de Madrid, Madrid.

García Toca, I., Nader Carreta, F. (2009). Estereotipos masculinos en la relación de pareja. Enseñanza e investigación en Psicología, 14(1), 37-45.

Gill, M., Roca, M. y García-Campayo, J. (2014). Crisis económica y salud mental. Informe SESPAS 2014. Gac Saint, 28(S1), 104-108.

Guillamón, N. (2003). Variables socioeconómicas y problemas interiorizados y exteriorizados en niños y adolescentes. (Tesis doctoral). Universidad autónoma de Barcelona, Bellaterra.

Pérez Padilla, J., Lorence Lara, B. y Menéndez Álvarez-Dardet, S. (2010). Estrés y competencia parental: Un estudio con padres y madres trabajadores. Suma psicológica, 17 (1), 47-57.

Urbanos-Garrido, R. M. y González, B. (2013). Desempleo y salud: Un análisis de la repercusión de la crisis económica sobre la salud de los españoles. Estudios de Economía Aplicada, 31(2), 303-326. 